\title{
Comparison of Esthetic Outcomes of Maxillary Lateral Incisor Agenesis Treatment by Orthodontic Space Closure Versus Implant Placement (Evaluated by Pink Esthetic Score)
}

\author{
Hedaiat moradpoor ${ }^{1}$, Farshad Rahimi ${ }^{2}$, Amin Golshah ${ }^{2}$, Narges Akbari ${ }^{3} \&$ Sahar Raissi $^{4}$ \\ ${ }^{1}$ Prosthodontics department, School of dentistry KermanshahUniversity of Medical Sciences, Iran \\ ${ }^{2}$ Orthodontics Department, School of Dentistry, Kermanshah University of Medical Sciences, Iran \\ ${ }^{3}$ General Dentist, School of dentistry, KermanshahUniversity of Medical Sciences, Iran \\ ${ }^{4}$ Prosthodontics department, School of dentistry, KermanshahUniversity of Medical Sciences, Iran \\ Correspondence: Sahar Raissi, Prosthodontics department, School of dentistry, KermanshahUniversity of Medical \\ Sciences, Iran.Email: dr.raisi97@gmail.com
}

Received: September 26, 2017

Accepted: October 22, 2018

Online Published: November 30, 2018

doi:10.5539/jmbr.v8n1p178

URL: https://doi.org/10.5539/jmbr.v8n1p178

\begin{abstract}
Introduction: Due to the fundamental role of esthetics in the outcomes of dental treatments, especially in the anterior region (esthetic zone), the necessity of considering the matter of esthetics in clinical studies has become into focus in the current era. The aim of this study was the evaluation of esthetic outcomes of two treatment protocols in the treatment of congenital uni-lateral missing of maxillary lateral incisors as well as patient satisfaction from the treatment outcomes.

Methods: in this study the sample size was 24 people (16 women and 8 men), These individuals sought dental treatment for replacement of the congenitally missing maxillary lateral incisor. Convenience sampling method was used and patients were divided into two groups regarding the kind of treatment they received. The two treatment protocols included: 1. Space closure by means of orthodontic treatment and then reshaping the canines; and 2. Space regaining by means of orthodontic treatment and replacing the lateral incisor with dental implants. Photographs of patients were acquired from the frontal view with retraction of the lips using digital cameras. Photographs were evaluated for Pink esthetic score.
\end{abstract}

Results: No significant difference was detected between the two study groups in the evaluated factors in this study.

Conclusion: The results of this study indicated that there is no significant difference in esthetic results in the two groups. Furthermore, both groups lead to similar results in patient satisfaction from treatment outcomes.

Keywords: Congenital missing of maxillary lateral incisors, esthetic evaluation, space closure, dental implants, patient satisfaction

\section{Introduction}

Tooth is considered as the most important physical characteristic for developing self-confidence (Hershon, 19870; Berscheid, 1972). Those who are satisfied with their face have a greater self-confidence compared to the individuals who are not satisfied with it (Berscheid, 1972; Albino, 1990). Congenital missing teeth in the anteroposterior regions can impair the balance and symmetry of smile and bringing about a negative effect on self-confidence (Cunningham, 2007; Van der Geld, 2007).

The treatment plan for replacing toothless space resulting from congenital missing maxillary lateral incisor includes removable partial denture, common fixed bridges, fixed cantilever prostheses, resin-bonded bridges, auto transplantation, displacing the canine tooth with the help orthodontics to close the toothless space and placing single-tooth implant (Rupp, 1997; Krassnig, 2011).

In the process of closing the space, the canine teeth should be displaced towards mesial and its morphology should change into lateral incisor. Further, the first premolar tooth should change into canine tooth. Since these teeth are different in terms of anatomy, color, and gum height, if the tooth replacement is not performed well, the 
static results of the treatment will be impaired (Rosa, 2007; Pini, 2012). If this process is well planned and performed based on an interdisciplinary approach, acceptable outcomes can be achieved (Krassnig, 2011; Zachrisson, 2007). The placed dental implants can also have satisfactory static results.

The present study has been conducted with the aim of comparing aesthetic consequences resulting from orthodontic closure of the space and opening the space and placing implant in the congenitally missing maxillary lateral incisor region. Furthermore, the extent of satisfaction of patients in these two treatment groups has been measured by a questionnaire.

\section{Methods}

The present study with the ethics code of IR.KUMS.REC.1396.344 has been approved by Kermanshah University of Medical Sciences, Iran. In this study, 24 patients with the history of congenital unilateral missing of lateral incisor treated by two different treatment methods of orthodontic closure of the space and recontouring the canine tooth as well as the treatment option of opening the space and placing implant were investigated.

Intraoral photography was prepared from frontal view using a retractor and with the help of a digital camera

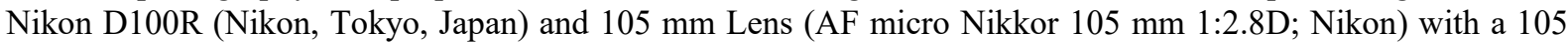
$\mathrm{mm}$ flash ring (Nikon Macro Speedlight SB-29S; Nikon). To assess the tooth in relation to the opposite tooth, the photographs were prepared considering the dental midline. However, for evaluating further details, photography was also done on every single lateral incisor replaced by the two methods. The photographs were prepared as printed and in the form of an electronic file. Evaluation of intraoral photographs was conducted by a dental prosthesis specialist and a student in the following framework:

Table 1. Assessment criteria - PES (PES: pink esthetic score)

\begin{tabular}{cccc}
\hline Parameter & Absent & Incomplete & Complete \\
\hline Mesial papilla & 0 & 1 & $\mathbf{2}$ \\
Distal papilla & 0 & 1 & $\mathbf{2}$ \\
& Major discrepancy & Minor Discrepancy & No Discrepancy \\
Curvature of facial mucosa & 0 & 1 & $\mathbf{2}$ \\
Level of facial mucosa & 0 & 1 & $\mathbf{2}$ \\
Root convexity, soft tissue color and & 0 & & $\mathbf{2}$ \\
texture & & & $\mathbf{1 0}$ \\
Maximum total PES score & & &
\end{tabular}

A score range of 0-2 should be assigned, where overall a score of 0-10 should be obtained from the measure (PES) (Lanza, 2017 and Cho, 2010). To reduce the bias and enhance the replicability of the results, each photography was measured twice and in two different days based on PES index.

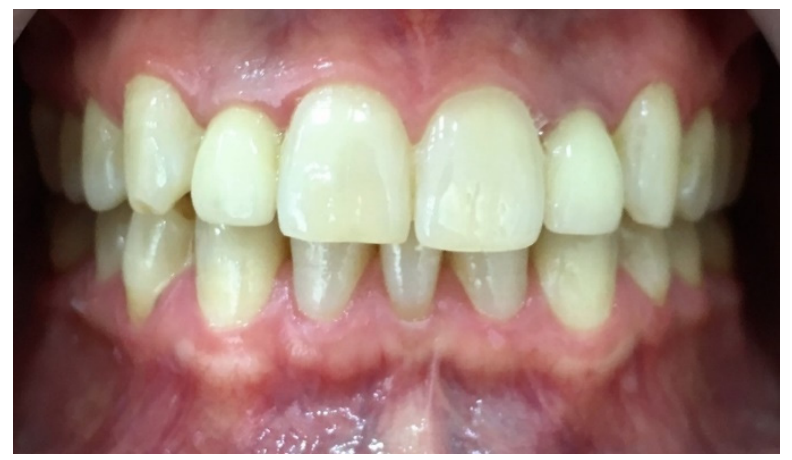

Figure 1. Frontal view of intraoral photography

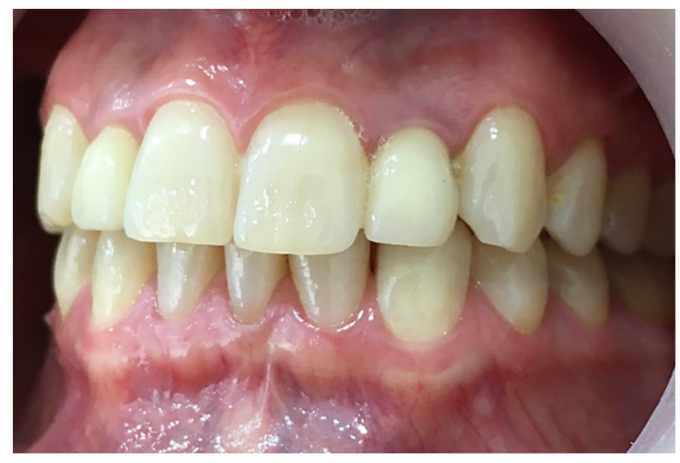

Figure 2. Intraoral photography to record the information related to maxillary lateral incisor 
After data collection, the information was analyzed by SPSS 18 (Inc., Chicago, IL, USA). The data collection method in this study was based on investigating the photographs prepared from the patients and scoring the issues involved in PES scoring (Lanza, 2017; Cho, 2010). These variables include the following:

Table 2

\begin{tabular}{lllllll}
\hline PES & Mesial & Distal & Curvature of facial & Level of facial & Root convexity, soft tissue color & Total PES \\
& papilla & papilla & mucosa & mucosa & and texture & (Max 10) \\
\hline
\end{tabular}

\section{Results}

In the present study, 24 individuals were present, out of whom 13 subjects (54.2\%) were in the implant group while 11 subjects $(45.8 \%)$ were in the canine reshaping group. The mean and standard deviation of the age of the subjects in this study was $26.08 \pm 2.84$. There was no significant relationship between gender and type of treatment (Fisher's Exact Test, $\mathrm{P}=1$ ).

there was a significant difference between the treatments in terms of Distal papilla variable $(\mathrm{P}=0.013)$. In this regard, the median and mean rank of this variable were lower in the group treated by implant placement, as compared with the group treated by space closure or canine recontouring. There was no significant difference in other variables (Table 3).

Table 3. Descriptive statistics for the PES subcategories

\begin{tabular}{|c|c|c|c|c|c|c|c|c|}
\hline & & Mean & $\begin{array}{l}\text { Standard } \\
\text { Deviation }\end{array}$ & Median & $\begin{array}{l}\text { Mean } \\
\text { Rank }\end{array}$ & $\begin{array}{c}\text { Percentile } \\
25\end{array}$ & $\begin{array}{c}\text { Percentile } \\
75\end{array}$ & P-value \\
\hline \multirow[t]{2}{*}{ Mesial papilla } & Implant & 1.77 & 0.44 & 2.00 & 12.23 & 2.00 & 2.00 & \multirow{2}{*}{.865} \\
\hline & Canine Reshape & 1.82 & 0.40 & 2.00 & 12.82 & 2.00 & 2.00 & \\
\hline \multirow[t]{2}{*}{ Distal papilla } & Implant & 1.08 & 0.49 & 1.00 & 9.23 & 1.00 & 1.00 & \multirow{2}{*}{.013} \\
\hline & Canine Reshape & 1.73 & 0.47 & 2.00 & 16.36 & 1.00 & 2.00 & \\
\hline \multirow{2}{*}{$\begin{array}{l}\text { Curvature of facial } \\
\text { mucosa }\end{array}$} & Implant & 1.38 & 0.65 & 1.00 & 10.00 & 1.00 & 2.00 & \multirow{2}{*}{.063} \\
\hline & Canine Reshape & 1.91 & 0.30 & 2.00 & 15.45 & 2.00 & 2.00 & \\
\hline \multirow{2}{*}{$\begin{array}{l}\text { Level of facial } \\
\text { mucosa }\end{array}$} & Implant & 1.23 & 0.83 & 1.00 & 13.65 & 1.00 & 2.00 & \multirow{2}{*}{.392} \\
\hline & Canine Reshape & 1.00 & 0.45 & 1.00 & 11.14 & 1.00 & 1.00 & \\
\hline \multirow{2}{*}{$\begin{array}{l}\text { Root convexity, soft } \\
\text { tissue color and } \\
\text { texture } \\
\text { a Mann-Whitney U }\end{array}$} & Implant & 1.69 & 0.63 & 2.00 & 14.00 & 2.00 & 2.00 & \multirow[b]{2}{*}{277} \\
\hline & Canine Reshape & 1.45 & 0.52 & 1.00 & 10.73 & 1.00 & 2.00 & \\
\hline
\end{tabular}

\section{Discussion}

Missing maxillary lateral incisor is the most common congenital missing of permanent teeth in the esthetic zone and accounts for around 20\% of dental anomalies (Robertsson, 2000 and Fekonja, 2005 and Kavadia, 2011). Considering the essential esthetic role in the outcomes resulting from dentistry treatments especially in esthetic zone, the necessity of dealing with esthetic issues in clinical research has attracted more attention.

This retrospective cohort study was conducted with this fundamental question whether the esthetic outcomes resulting from treating congenital missing of maxillary lateral incisor by opening the space and replacing with implant and space closure and reshaping the canine teeth are significantly different. The results of the study suggested absence of any significant difference between the esthetic consequences resulting from the two mentioned treatment methods.

The results of the present research indicated that the mean Total PES is 7.15 in the implant group, which was partially in line with results obtained by C Mangano et al (Mangano, 2014). In their study, the mean PES was $8.15 \pm 1.69$, which did not change significantly during a three-year follow-up.

There was no significant difference in Total PES between the two groups: 1. Treated by orthodontic closure of the space and canine recontouring, and 2. Opening the space or keeping the space and replacing congenitally missing maxillary lateral incisor with implant $(\mathrm{P}=0.328)$. In a systematic review conducted in 2016 by Kiliaridis et al (Kiliaridis, 2016), the treatment plans for treating congenitally missing lateral incisors were evaluated. In 
this study, the papers published from January 1975 to March 2015 were assessed, where five papers had directly dealt with comparison of these two methods. Nevertheless, based on the study by Kiliaridis it seems that space closure via orthodontics if possible is more effective psychologically since it gives the feeling to the patient that the set of dentition belongs to them and does not require prosthesis.

Björn U. Zachrisson et al (Zachrisson, 2011) stated that through space closure by orthodontics and placing porcelain veneers of several teeth, the treatment outcomes can approach natural dentition as much as possible. Furthermore, the advantages of this method were considered as follows: 1) young patients receive the final treatment outcomes during teenage years 2) the general treatment plan can be completed after the orthodontics process 3 ) long-term harmony of the tooth and its surrounding structures will have a natural appearance. Thus, they preferred the reasons of orthodontic space closure and canine reshaping to opening the space or preserving it.

In the present study, since the mean age of the patients was $26.08 \pm 2.84$, there was no limitation for implant placement. It can be stated that the negative effect of young age in developing time constraint for receiving implant treatment has not been influential in the comparison of the two methods in the view of patients.

In studies conducted by PC Armbruster et al (Armbruster, 2005) the judgment of 140 dentists, 43 orthodontists, 29 specialists of other fields, and 40 normal individuals was measured in relation to the attractiveness of the smile of people with congenital missing of maxillary lateral incisor through photography. The photographs included: resin-bonded bridges, implant placement and space closure through displacing the canine tooth. The cases without congenitally missing maxillary lateral incisor were used as control. The results showed that the normal people chose the photographs related to displaced canines as the best esthetic cases. According to orthodontists, the scoring order from the worst to best cases was: cases without missing, space closure and displacing the canine teeth, resin-bonded bridges, and implant. The specialists of other fields and general dentists, unlike the orthodontists, preferred implant due to static reasons.

Note that the present study had some limitations. Among the factors affecting the statics of esthetic and prosthetic treatments are gum color and healthy gum with no inflammation, which has not been considered in PES criterion. Possibly, if this factor is involved in the assessment of static consequences resulting from the treatments, the obtained results can have more significant differences.

\section{Conclusion}

This study indicated that there was no significant difference between the esthetic consequences resulting from treating congenitally missing maxillary lateral incisor by opening the space and implant placement along with space closure and reshaping canine tooth based on PES criterion. The only significant difference existed between the two treatments in terms of Distal papilla, where the ranked mean and median in the group treated by implant placement was lower than that of the group treated by space closure and canine reshaping. There was no other significant difference among the other variables.

\section{References}

Albino, J. E., Alley, T. R., Tedesco, L. A., Tobiasen, J. A., Kiyak, H. A., \& Lawrence, S. D. (1990). Esthetic issues in behavioral dentistry. Annals of Behavioral Medicine, 12(4), 148-155.

Armbruster, P. C., Gardiner, D. M., Whitley, J. B., \& Flerra, J. (2005). The congenitally missing maxillary lateral incisor. Part 1: esthetic judgment of treatment options. World Journal of Orthodontics, 6(4).

Berscheid, E., Walster, E., \& Bohrnstedt, G. (1972). Body image: a Psychology Today questionnaire. Psychology Today, 6(2), 57-64.

Cho, H. L., Lee, J. K., Um, H. S., \& Chang, B. S. (2010). Esthetic evaluation of maxillary single-tooth implants in the esthetic zone. Journal of Periodontal \& Implant Science, 40(4), 188-193.

Cunningham, S. J., \& O’Brien, C. (2007, June). Quality of life and orthodontics. In Seminars in Orthodontics (Vol. 13, No. 2, pp. 96-103). WB Saunders.

Fekonja, A. (2005). Hypodontia in orthodontically treated children. The European Journal of Orthodontics, 27(5), 457-460.

Hershon, L. E., \& Giddon, D. B. (1980). Determinants of facial profile self-perception. American Journal of Orthodontics, 78(3), 279-295. 
Kavadia, S., Papadiochou, S., Papadiochos, I., \& Zafiriadis, L. (2011). Agenesis of maxillary lateral incisors: a global overview of the clinical problem. ORTHODONTICS: The Art \& Practice of Dentofacial Enhancement, 12(4).

Kiliaridis, S., Sidira, M., Kirmanidou, Y., \& Michalakis, K. (2016). Treatment options for congenitally missing lateral incisors. Eur J Oral Implantol, 9, S5-S24.

Krassnig, M., \& Fickl, S. (2011). Congenitally missing lateral incisors-a comparison between restorative, implant, and orthodontic approaches. Dental Clinics, 55(2), 283-299.

Lanza, A., Di Francesco, F., De Marco, G., Femiano, F., \& Itro, A. (2017). Clinical application of the PES/WES Index on natural teeth: Case report and literature review. Case reports in dentistry, 2017.

Mangano, C., Levrini, L., Mangano, A., Mangano, F., Macchi, A., \& Caprioglio, A. (2014). Esthetic evaluation of implants placed after orthodontic treatment in patients with congenitally missing lateral incisors. Journal of Esthetic and Restorative Dentistry, 26(1), 61-71.

Pini, N. I. P., De-Marchi, L. M., Gribel, B. F., Ramos, A. L., Furquim, L. Z., \& Pascotto, R. C. (2012). Analysis of width/height ratio and gingival zenith in patients with bilateral agenesis of maxillary lateral incisor. Dental Press Journal of Orthodontics, 17(5), 87-93.

Robertsson, S., \& Mohlin, B. (2000). The congenitally missing upper lateral incisor. A retrospective study of orthodontic space closure versus restorative treatment. The European Journal of Orthodontics, 22(6), 697-710.

Rosa, M., \& Zachrisson, B. U. (2007). Integrating space closure and esthetic dentistry in patients with missing maxillary lateral incisors. Journal of clinical orthodontics: JCO, 41(9), 563.

Rupp, R. P., Dillehay, J. K., \& Squire, C. F. (1997). Orthodontics, prosthodontics, and periodontics: a multidisciplinary approach. General dentistry, 45(3), 286-289.

Van der Geld, P., Oosterveld, P., Van Heck, G., \& Kuijpers-Jagtman, A. M. (2007). Smile attractiveness: self-perception and influence on personality. The Angle Orthodontist, 77(5), 759-765.

Zachrisson, B. U. (2007). Improving the esthetic outcome of canine substitution for missing maxillary lateral incisors. World journal of orthodontics, 8(1), 72.

Zachrisson, B. U., Rosa, M., \& Toreskog, S. (2011). Congenitally missing maxillary lateral incisors: canine substitution. American journal of orthodontics and dentofacial orthopedics, 139(4), 434.

\section{Copyrights}

Copyright for this article is retained by the author(s), with first publication rights granted to the journal.

This is an open-access article distributed under the terms and conditions of the Creative Commons Attribution license (http://creativecommons.org/licenses/by/4.0/). 